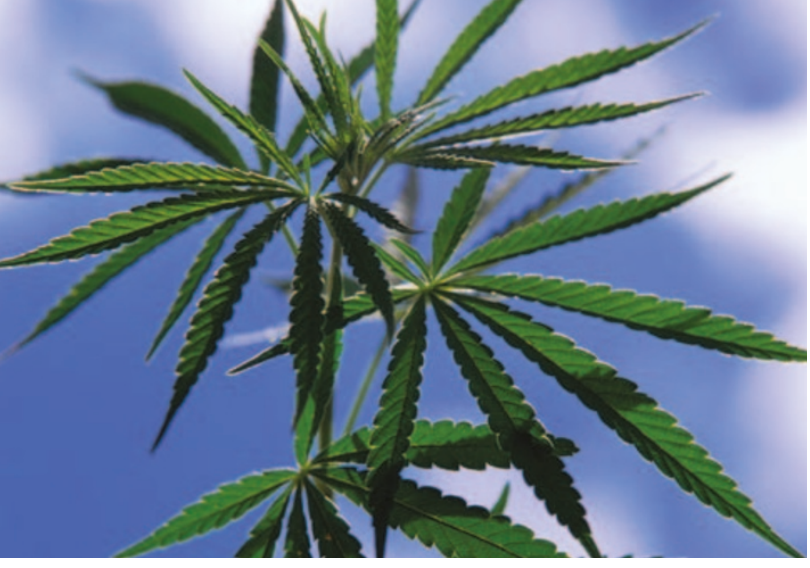

LEARNING AND MEMORY

\section{Accelerated ageing}

A decline in learning and memory frequently accompanies the normal ageing process as well as neurodegenerative disorders. However, the molecular mechanisms that underlie cognitive deficits that arise during ageing are largely uncharacterized. Bilkei-Gorzo and colleagues have recently revealed an unexpected role for the endocannabinoid system in learning and memory decline with increasing age.

Short-term exposure to cannabinoids is well known to have a negative effect on various aspects of learning and memory, and acute pharmacological blockade of the cannabinoid CB1 receptor prevents memory deficits induced by cannabinoids, enhances memory when administered in isolation and decreases memory deficits in older animals. However, the effects of longterm alterations of the endocannabinoid system on learning and memory were, until now, unknown.

Bilkei-Gorzo and colleagues investigated the effects of deleting the CB1 receptor gene Cnr1 on three tests of learning and memory - the rotarod test, which measures psychomotor skill learning; an operant conditioning paradigm, which indexes working memory, procedural learning and spatial memory; and a social recognition test - in mice of different ages. Young mice (6-7 weeks old) that lacked the Cnrl gene performed as well as or even better than did wild-type mice on these tasks. By contrast, absence of this gene in mature mice (3-5 months old) led to striking impairments in all three measures of learning and memory compared with their age-matched controls. Their deficits were on a par with the learning and memory abilities of aged mice (14-17 months old), which were typically impaired on these tasks relative to the younger control groups, indicating that memory decline is accelerated in the absence of $\mathrm{CB} 1$ receptors.

Intriguingly, these impairments were correlated with neuronal loss in areas CA1 and CA3 of the hippocampus. Bilkei-Gorzo and colleagues therefore suggested that the endocannabinoid system has an important neuroprotective effect that hinges on the presence of $\mathrm{CB} 1$ receptors.

With an increasing ageing population, there is a growing need to develop novel ways to treat or prevent cognitive deficits. This work provides encouraging signs that the endocannabinoid system could be a potential therapeutic target for the treatment of cognitive decline during the course of ageing.

Alison Rowan

\section{(1) References and links}

ORIGINAL RESEARCh PAPER Bilkei-Gorzo, A. et al. Early age-related cognitive impairment in mice lacking cannabinoid CB1 receptors. Proc. Natl Acad. Sci. USA 102 15670-15675 (2005)

FURTHER READING Hedden, T. \& Gabrieli, J. D. E. Insights into the ageing mind: a view from cognitive neuroscience. Nature Rev. Neurosci. 5, 87-96 (2004)

\title{
The moody barrier
}

The blood-brain barrier (BBB), a selective physical and chemical barrier that insulates the nervous system from the body fluid, is crucial for maintaining a stable ionic environment and ensuring appropriate firing of neurons. Two studies, published in Cell, show that moody, a gene that encodes a newly identified G-protein-coupled receptor (GPCR), is necessary for the formation and maintenance of the fly BBB.

In a reverse genetic screen for factors with glial expression and function, Schwabe and colleagues identified two GPCRs, Moody and Tre1, as well as a regulator of G-protein signalling, Loco. Moody, Loco and the G-protein subunits Goi and Goo are expressed in the surface glia - the thin layer of epithelial cells that constitutes the fly BBB. Loco colocalizes with Moody at the plasma membrane, and physically interacts with $\mathrm{G} \alpha \mathrm{i}$ and $\mathrm{G} \alpha \mathrm{o}$, which indicates that the four proteins are part of a common signalling pathway. When the expression of any of these factors is abolished in fly embryos using RNA interference, the $\mathrm{BBB}$ development is significantly compromised. In the case of moody, complete loss of function results in embryonic lethality due to defective insulation of the nervous system.

In the second study, Bainton and colleagues identified moody in a genetic screen for mutants with altered cocaine sensitivity. moody encodes two proteins, Moody- $\alpha$ and $-\beta$, which differ in their long carboxy-terminal domains. Both forms of Moody are expressed in the surface glia and at regions of cell-cell contact. When the expression of moody is transiently inhibited in adult flies, the BBB is disrupted in a reversible manner, which indicates that Moody is continuously required to insulate the nervous system. Interestingly, the role of Moody in drug sensitivity can be dissociated from its role in nervous system insulation, whereas either Moody- $\alpha$ or $-\beta$ is sufficient for $\mathrm{BBB}$ formation, both protein forms are necessary for flies to show normal cocaine sensitivity.

As GPCRs and other newly identified molecules are important for the BBB in both flies and mammals, studies of the fly BBB could provide important insights into how the mammalian BBB is formed and regulated, and how this might lead to changes in drug sensitivities and other complex behaviours.

Jane Qiu

\section{(2) References and links} ORIGINAL RESEARCH PAPERS Schwabe, T. et al. GPCR signaling is required for blood-brain barrier formation in Drosophila. Cell 123, 133-144 (2005) | Bainton, R. J. et al. moody encodes two GPCRs that regulate cocaine behaviors and blood-brain barrier permeability in Drosophila. Cell 123, 145-156 (2005)

FURTHER READING Abbott, N. J. et al. Astrocyte-endothelial interactions at the bloodbrain barrier. Nature Rev. Neurosci. (in the press) 